\title{
Índices de libros recensionados
}

Agudo Romeo, $\mathrm{M}^{\mathrm{a}}$ del M., Encuentra Ortega, A. y Esteban Lorente, J. F. (editores), Juan de Horozco y Covarrubias de Leyva. Trescientos emblemas morales.

AlPERs, C., A Politics of Grace. Hope for redemption in a post-Christendom context.

Álvarez de los Mozos, P., Servir a los pobres, promover la justicia. Panorámica histórica del apostolado social de la Compañía de Jesús.

Angelini, A. y Pellegrini, M. (editores), La Chiesa di san Vigilio a Siena. Storia e arte. Dalle origini monastiche allo splendore dell'età barocca.

Arias de SaAvedra Alías, I., Jiménez Pablo, E. y López-Guadalupe MuÑoZ, M. L. (editores), Subir a los altares: modelos de santidad en la Monarquía Hispánica (siglos XVI-XVIII).

Barentsen, J., van Den Heuvel, S. C. y Lin, P. (editores), The end of leadership? Leadership and authority at crossroads.

Begheyn, P., Jesuit Books in the Dutch Republic and its Generality Lands 1567-1773.

Belcher, W. L., The Jesuits in Ethiopia (1609-1641): Latin Letters in Translation.

Belzunegui Eraso, A. (director), L'Església Ortodoxa Romanesa a Catalunya. Estructura de relacions $i$ comunitat de creients.

Belzunegui Eraso, A., Sánchez Cervelló, J. y Reig Tapia, A. (coordinadores) Església i franquisme. De la col-laboració amb el franquisme al seu combat.

Benci, F. (introducción, traducción y comentario de Paul Gwynne) Francesco Benci's Quinque martyres.

BERTONE, T., I miei papi.

Bobonich, C. (editor), The Cambridge Companion to Ancient ethics.

Borghesi, M., Jorge Mario Bergoglio. Una biografía intelectual. Dialéctica y mística.

Te BraKe, W. P., Religious war and religious peace in Early Modern Europe.

Bruna, A., L'accord d'Angora de 1921. Théâtre des relations franco-kémalistes et du destin de la Cilicie.

Callado Estela, E. (editora), La catedral barroca. Iglesia, sociedad y cultura en la Valencia del siglo XVII (vol. I).

Camarero Calandria, E. y Cortés Martínez, F. (coordinadores), Amor a lo visible. Tras las huellas de la Compañía de Jesús en Córdoba (catálogo de la exposición).

CÁrCEl OrTí, Ma. M., Diplomática episcopal.

CÁrcel Ortí, V. (editor) La II República y la Guerra Civil en el Archivo Secreto Vaticano (VI. Documentos del año 1938).

Cárcel Ortí, V., 1936. El Vaticano y España.

Carretero Calvo, R., Historia Domus Turiasonensis. El relato histórico del colegio de la Compañía de Jesús de Tarazona (1591-1628). 
Casas Hernández, M., Escenografía Barroca. El tabernáculo de los Churriguera de la Catedral Nueva de Salamanca.

602

Chantre, B., Les derniers jours de René Girard.

Cheza, M., Martínez Saavedra, L. y Sauvage, P. (con la colaboración de Rocha de Souza, A. y SAPPIA, C.), Dictionnaire historique de la théologie de la libération (seguido de de Genèse, évolution et actualité de la théologie de la libération).

Chrétiens d'Orient. 2000 ans d'histoire.

Claveria Nadal, M. (editor), Viri antiqui.

Clément, O. N., El rostro interior.

584

CONGREGAZIONE PER LA DOTTRINA DELla FEDE, Nota dottrinale circa alcune questioni riguardanti l'impegno e il comportamento dei cattolici nella vita política (21-XI-2002).

Costa, G. y Foglizzo, P. (editores), Il lavoro è dignità. Le parole di Papa Francesco.

Dagnino R. y GrazI, A. (editores), Believers in the nation. European Religious Minorities in the Age of Nationalism (1815-1914).

Dahan, A. G. y Rillon-Marne, Z. (coordinadores), Philippe le Chancelier prédicateur, théologien et poète parisien du début du XIII I ${ }^{\mathrm{me}}$ siècle.

Delcorno, P. (editor), Politiche di misericordia tra teoria e prassi. Confraternite, ospedali e Monti di Pietà (XIII-XVI secolo).

Diotallevi, L., Fine corsa. La crisi del cristianesimo comme religione confessionale.

Dive, B., John Henry Newman and the imagination.

DREHER, R. The Benedict Option. A strategy for Christians in a post-Christian nation / DREHER, R., La opción benedictina. Una estrategia para los cristianos en una sociedad postcristiana.

Drewermann, E., Gestalten des Bösen.

Dodson, J. R. y Briones, D., Paul and Seneca in Dialogue.

Duch, Ll., Sortida del laberint. Una trajectoria intel-lectual: lliçons Ferrater Mora.

Dupont, A., Boodts, S., Partoens, G. y Leemans, J. (editores), Preaching in the Patristic Era. Sermons, Preachers, and Audiences in the Latin West.

Escrivá de Balaguer, J. (edición crítico-histórica de L. Cano, F. Castells y J. A. LOARTE), En diálogo con el Señor. Textos de la predicación oral. Escrivá de BALAGUER, J., Escritos varios (1927-1974).

Fehrenbach, J., Von Galen. Un évêque contre Hitler.

Felipo Orts, A. y Callado Estela, E., Entre la cátedra y el púlpito. Los pavordes de la Universidad de Valencia (Siglos XVI-XVII).

FerLan, C., Sbornie sacre, sbornie profane. L'ubriachezza dal Vecchio al Nuovo Mondo.

Fernández SAlvador, C., Encuentros y desencuentros con la frontera imperial. La iglesia de la Compañía de Jesús de Quito y la misión en el Amazonas (siglo XVII). 
Fidalgo, J. M., Lázaro, R. y Caballero, J. L., Son tus huellas el camino. Reflexiones sobre vocación y libertad.

François, W., DEn Hollander, A. y AgTen, E. (editores), Vernacular Bible and religious reform in the Middle Ages and Early Modern Era.

Fris-Larrouy, V., D'un soleil à l'autre. Jean-Denis Attiret, missionnaire jésuite peintre de l'Empereur de Chine.

Fuertes, J. L., LÁzaro, M. y Zorroza [Huarte], Ma . I. (editores), Mística y filosofía en el Siglo de Oro.

Fumagalli, A., Camminare nell'amore. La teologia morale di Papa Francesco.

Funguerio, J., Libro sobre la buena enseñanza y educación de los jóvenes (1584). De puerorum disciplina et recta educatione liber.

García de Cortázar, F., Católicos en tiempos de confusión.

García Pérez, F. J., La cruzada antilulista. El obispo Juan Díaz de la Guerra y la persecución del culto a Ramón Llull en la Mallorca del siglo XVIII.

García Vilardell, Ma . R., Martínez-Carbonell, A. y Vilarroig Martín, J., Religión, cultura y valores.

Gesché, A., La teología.

Gianotto, C., I Vangeli Apocrifi.

Gil Ambrona, A., Ignacio de Loyola y las mujeres. Benefactoras, jesuitas $y$ fundadoras.

González Gullón, J. L., DYA. La Academia y Residencia en la historia del Opus Dei (1933-1939).

González Marcos, I. y Sciberras, J. (editores), Vita quotidiana e tradizioni nei conventi dell'ordine di sant'Agostino.

González Velasco, M., Beatos José Agustín Fariña y Pedro de la Varga, de Valladolid, Agustinos. Mártires en Paracuellos.

González Velasco, M., Seis agustinos de La Valdavia, (Palencia) mártires en Paracuellos. Beatos Heliodoro Merino Merino, Jesús Largo Manrique, Dionisio Terceño Vicente, José Noriega González, Francisco Fuente Puebla y Román Martín Mata.

González-Mohíno Bartolomé, F., La humanidad de Cristo, causa de la gracia, causa de la Iglesia, en l'Église du Verbe Incarné de Charles Journet.

Goris, H. y Scноот, H. (editores), The Virtuous Life. Thomas Aquinas on the Theological Nature of Moral Virtues.

Goulding, G. K., A Church of Passion and Hope. The Formation of an Ecclesial Disposition from Ignatius Loyola to Pope Francis and the New Evangelization.

Grieu, É., Rimbaut, G. y Blanchon, L., (directores) Qu'est-ce qui fait vivre encore quand tout s'écroule? Une théologie à l'école des plus pauvres.

Guardini, R., El Señor. Meditaciones sobre la persona y la vida de Jesucristo. 
Gutiérrez García, S., López Martínez-Morás, S. y Pérez Barcalá, G. (editores), El culto jacobeo y la peregrinación a Santiago a finales de la Edad Media. Crisis y renovación.

Hernández Martínez, J. M., Claret y el protestantismo de su tiempo. La utopía de un encuentro imposible.

Himes, K. R. et alii (editores), Modern Catholic Social Teaching: Commentaries and Interpretations.

Hódar Maldonado, M., San Juan de la Cruz. Cántico Espiritual - Todo Amor.

HoREMANs, K., La relation entre «pacte» et «tabou» dans le discours autobiographique en France (1750-1850).

IgNACIO DE LOYOLA, Ejercicios espirituales. Para mejor comprenderlos y practicarlos (versión actual en paralelo).

Kann, Ch., Loewe, B., Rode, Ch. y Uckerman, S. L. (editores), Modern Views of Medieval Logic.

KeRn, U., Der transzendentale Aufklärer Meister Eckhart.

KeYt, D., Nature and Justice. Studies in the Ethical and Political Philosophy of Plato and Aristotle.

KIENZLER, K., Glauben - Wie geht das? Phänomenologie des Glaubens .

TEN Klooster, A., Thomas Aquinas on the beatitudes. Reading Matthew, Disputing Grace and Virtue, Preaching Happiness.

Knutsen, G. W., Los procesos por superstición en la Inquisición en Barcelona y Valencia, 1478-1700: siervos de Satanás o maestros de demonios.

KowalczyK, D. (editor), La Parola nelle parole.

La Parra López, E., Fernando VII. Un rey deseado y detestado.

LazCano, R., Tesauro Agustiniano. Vida, obra y bibliografía de escritores, (...) agustinos/as y agustinos/as recoletos/as de España, Portugal, América Latina y Filipinas.

Leland SAAR, E., Luther and the Reformation of the later Middle Ages. 534

LOARTE, J. A., A Jesús por María. Escenas Marianas.

LoHFink, G., Am Ende Das Nichts? Über Auferstehung und ewiges Leben.

López Alcaide, C., Puig Montada, J. y Roche Arnas P. (editores), Legitimation of Political Power in Medieval Thought.

495

de Luis Vizcaíno, P., El Monacato de san Agustín. Comunión, comunidad, ministerio.

Luomanen, P., Pessi, A. B. y Pyysiärnen, I. (editores), Christianity and the Roots of Morality. Philosophical, Early Christian and Empirical Perspectives.

Marchetto, A., "La reforma e le riforme nella Chiesa" (Una risposta).

Marchetto, A. y Parise, G., Riforma a cinquanta anni dal Concilio Vaticano II su Presbyterorum Ordinis $e$ Optatam Totius per la formazione sacerdotale e un fecondo ministero presbiterale.

Marengo, G., La nascita di un'enciclica. Humanae vitae alla luce degli Archivi Vaticani. 
Martínez Lorca, Andrés (ed.), La filosofía en Al Ándalus.

Martínez Camino, J. A. (editor), Victimas y mártires. Aproximación histórica y teológica al siglo $X X$.

Mascarell Dauder, R., Epistolario Alfons Roig - María Zambrano (1955-1985).

Matteo, A. (editor), Il discernimento. "Questo tempo non sapete valutarlo?» $($ Lc 12,56).

Mele, A. R., Intenciones afectivas. El poder de la voluntad consciente.

Menozzi, D., I papi e il moderno. Una lettura del cattolicesimo contemporaneo (1903-2016).

Meynet, R., Le Psautier. Cinquième livre (Ps 107-150).

Miceviciute, J., Las 17 ciudades de Santa Teresa: libro de viajes.

Mieth, D. et alii (editores), Meister Eckhart in Paris and Strasbourg.

Minch, D., Eschatological Hermeneutics. The Theological Core of Experience and Our Hope for Salvation.

Minutelli, M., L'Arca di Saba: “i sereni animali che avvicinano a Dio”.

Montero [García], F., De la Cueva, J. y LouzaO, J. (editores), La historia religiosa de la España contemporánea: Balance y perspectivas.

Montero García, F., El movimiento católico en España. 1889-1936.

Moreno, D., Casiodoro de Reina. Libertad y tolerancia en la Europa del siglo XVI.

Los mozárabes. Historia, cultura y religión de los cristianos de Al-Andalus.

MusARRA, A., Il crepuscolo della crociata. L'Occidente e la perdita della Terrasanta.

Ortega Mentxaka, E., Ad maiorem Dei gloriam. La iconografía jesuítica en la antigua provincia de Loyola (1551-1767).

PANIKKAR, R., Obras completas.

PARRA, J. D., Claves de simbología. Las figuras esenciales de la ciencia de los símbolos y su relación con la cultura hispánica.

Pellitero Iglesias, R., Teología de la misión.

Pérez Chico, D. (coordinador), Cuestiones de la filosofía del lenguaje.

Pérez Gómez, Á. A. (versión actual en paralelo de) IGNACio de Loyola, Ejercicios Espirituales. Para mejor comprenderlos y practicarlos.

Pitaud, B., Jean-Jacques Olier (1608-1657).

Preston, P., España en la guerra civil europea. Contribuciones de un hispanista.

Quirós Rosado, R., Monarquía de Oriente. La corte de Carlos III y el gobierno de Italia durante la guerra de Sucesión española.

Rabaté, J.-C. y Rabaté, C., En el torbellino. Unamuno en la Guerra Civil.

Ravé Prieto, J. L., San Luis de los Franceses.

ReED, E. D., The Limit of Responsibility. Dietrich Bonhoeffer's Ethics for a Globalizing Er.

Revel, J.-F., Mémoires.

Rivera De Rosales, J., (director), La actualidad del padre Juan de Mariana. Rodríguez CuzA, F. E., La inquietud del tiempo. El diputado Félix Varela Morales en las Cortes españolas (1821-1823). 
Romizi, F., El Dios en la maleta. Los caminos de la significación mítica de los ecuatorianos católicos en Barcelona y Nueva York.

Rosa, M. y al Kalak, M. (editores), Lodovico Antonio Muratori. Religione e politica nel Settecento.

Ruiz Povedano, J. M., Málaga, de musulmana a cristiana. La transformación de la ciudad a finales de la Edad Media.

Sáenz Ruiz de Olalde, J. L., Historia de la Abadía de San Millán de la Cogolla (Siglos XV-XIX).

Sainz Bariáin, I., Poder, fasto y teatro: la «Comedia de san Francisco de Borja (1640)» de Matías Bocanegra, en su contexto festivo.

Sánchez Herrero, J., Historia de las Diócesis Españolas. Tomo 21. Iglesias de Astorga y Zamora.

SÁnchez Herrero, J. (coordinador), San Isidoro de Sevilla en Sevilla.

SCANnone, J. C., Il Vangelo della misericordia nello spirito del discernimento. L'etica sociale di Papa Francesco.

SchJørRing, J. H. y HJelm, N. A., History of Global Christianity (3 volúmenes).

Schröter, J., Jesus von Nazaret. Jude aus Galilea-Retter der Welt.

Scola, A., Familia, recurso decisivo.

SerRa Estellés, X., Inventari dels arxius parroquials de la Safor.

615

600

Serrano García, R., de Prado Moura, A. y Larriba, E. (editores), Dimensiones religiosas de la Europa del Sur (1800-1875).

SesboüÉ, B., L'acte théologique d'Irénée de Lyon à Karl Rahner. Les grandes créations en théologie chrétienne.

SEsÉ, J., En intimidad de amor con Dios. Reflexiones sobre la filiación divina y la vida espiritual, desde la enseñanza de los santos.

Somavilla RodríGuez, E., Los jóvenes la fe y el discernimiento vocacional.

Sommet, J., La condizione disumana. Un jesuita a Dachau, Bolonia (Italia) Dehoniane, 2017, 75 pp. [978-88-10-56759-3].

Soto ArtuñEDo, W., Alonso de Barzana, SJ (1530-1597), el Javier de las Indias Occidentales.

Soto ArtuñEdo, W., Diego de Pantoja, SJ (1571-1618). Un puente con la China de los Ming.

Steigerwald, G., Die frühchristlichen mosaiken des triumphbogens von S. Maria Maggiore in Rom.

Strong, D., A Call to Mission. The Jesuits in China. The French Romance. Vol. I: 1842-1955; vol. II: 1827-1957.

Teitler, H. C., The last pagan emperor. Julian the Apostate and the War against Christianity.

Theobald, Ch., Urgences pastorals du moment présent. Comprendre. Partager. Réformer.

Tras las huellas de Dios en la historia. Cuadernos de viaje de peregrinos a Tierra Santa.

VANNIER, M.-A. (directora) Judaïsme et christianisme chez les Pères.

Vidal i Quintero, M. (editora) Reforma y reformas en la Iglesia. Miradas críticas de las mujeres cristianas. 
Vila Despujol, I., La Compañía de Jesús en Barcelona (1600-1659). El colegio de Nuestra Señora de Belén se consolida.

Vilarroig Martín, J. y Cano Alarcón, M. J., Iglesia, sacramento y moral.

VInCENT, B., L'Islam d'Espagne au XVI ${ }^{\mathrm{me}}$ siècle. Résistances identitaires des morisques.

de Vitoria, F. (edición de Sarmiento Franco, A. y Zorroza Huarte, Ma

I.), Comentarios a la Prima Secundae de la Summa Theologiae de santo Tomás. Tomos I: De beatitudine / Sobre la felicidad (qq. 1-5) y III: De passionibus, habitibus et virtutibus / Sobre las pasiones, los hábitos y las virtudes (qq. 22-67).

WerBicK, J., Gott-menschlich. Elementar Christologie.

Wojtulewicz, Сн. M., Meister Eckhart on the Principle.

Yang, F. y Pettit, J. E. E., Atlas of Religion in China. Social and Geographical Contexts.

ZAS FrIZ DE CoL, R., Saggi ignaziani. 